Results Participants landed with more trunk flexion (success: $21.8^{\circ} \pm 13.2^{\circ}$; failed: $\left.30.3 \pm 17.2^{\circ}\right)(p<0.05)$, more anterior pelvic tilt (success: $4.2^{\circ} \pm 5.4$; failed: $7.7^{\circ} \pm 5.1^{\circ}$ ), and less lateral pelvic tilt towards the landing leg (success: $4.7 \pm 3.0^{\circ}$; failed: $2.7 \pm 3.7)$ during failed landings $(p<0.05)$. Higher rectus femoris, biceps femoris and gluteus medius excitation amplitudes were also observed during the failed landings $(p<0.05)$. MACD analysis identified that differences between failed and successful landings were initiated during the preparatory phase of the drop-jump.

Conclusions While biomechanical variables were significantly different during the landing phase, our novel MACD analysis identified that these differences initiated during the flight phase. Our findings also highlight that proximal joints play a critical role for landing successfully. Preventive training must therefore include how athletes prepare for a landing with a strong emphasis on upper body and proximal joint control.

\section{DOES HIP STRENGTH PREDICT DYNAMIC VALGUS IN FEMALE RECREATIONAL RUNNERS?}

1,2Gabriel Zeitoune, ${ }^{2} J u r a n d i r$ Nadal, ${ }^{3}$ Luiz Alberto Batista, 'Leonardo Metsavaht, ${ }^{4}$ Paulo Lucareli, 'Gustavo Leporace. 'Institute Brazil of Technologies in Health, RJ, Brazil; ${ }^{2}$ Biomedical Engineering Program, Federal University of Rio de Janeiro, RJ, Brazil; ${ }^{3}$ Biomechanics and Motor Behaviour Laboratory, State University of Rio de Janeiro, RJ, Brazil; ${ }^{4}$ Post Graduation Program of Rehabilitation Sciences, SP, Brazil

\subsection{6/bjsports-2021-IOC.310}

Background Dynamic valgus has been the focus of many studies to identify its association to an increased risk of runningrelated injuries. Many therapists suggest gluteus strengthening to address this movement dysfunction. However, it is not known which hip strength associated with this movement dysfunction.

Objective To test the correlation between hip strength and dynamic valgus in female runners.

Design Cross-sectional study.

Setting Biomechanics laboratory.

Participants Twenty-nine healthy recreational female runners.

Interventions Participants ran on a treadmill at $2.92 \mathrm{~m} / \mathrm{s}$. Kinematic data were collected using an 8 high-speed cameras motion analysis system (Vicon, Oxford, UK) with a sample rate of $250 \mathrm{~Hz}$. Maximal isometric hip strength was tested using a hand-held dynamometer.

Main Outcome Measurements Predictor variables were peak isometric strength of hip extensors, abductors and external rotators. Interest variables were contralateral pelvic drop, hip adduction and internal rotation (peak angles and joint excursion) during stance phase of running. Association between predictors and interest variables were tested using Pearson Correlation Coefficient (alpha $=0.05$ ).

Results There was no significant correlation between hip strength and contralateral pelvic drop ( $\mathrm{r}$ ranging from -0.09 to $0.32, \mathrm{p}>0.05$ ), hip adduction ( $\mathrm{r}$ ranging from -0.23 to $0.11, \mathrm{p}>0.05)$, and hip internal rotation ( $\mathrm{r}$ ranging from 0.33 to $0.01, \mathrm{p}>0.05)$.

Conclusions Although previous studies showed dynamic valgus was associated to hip weakness during single-leg squat and jump-landing tasks, the results of our study suggested that caution should be taken when linking hip disorders in female runners during running to posterolateral hip strength. These findings could be related to the linear nature of the statistical methods used to predict the biomechanical dysfunctions. It might be necessary to apply more robust techniques, as Artificial Neural Networks and Random Forests, to understand how physical variables interact to predict dynamic valgus in runners.

\section{SHORT TRACK VS HOCKEY HELMETS: INVESTIGATING IMPACT ATTENUATION PROPERTIES OF HELMETS IN TWO SKATING SPORTS}

${ }^{1}$ Daniel Aponte, ${ }^{2}$ Suzanne Leclerc, ${ }^{1}$ David Pearsall. 'McGill University, Montreal, Canada; ${ }^{2}$ Institut National du Sport du Québec, Montreal, Canada

\subsection{6/bjsports-2021-IOC.311}

Background Certification standards governing short track (ST) helmets only require high velocity impacts be tested. Rotational acceleration and low velocity impacts are mechanisms of injury which are known to cause concussion. Conversely, ice hockey (IH) helmet certification require low velocity impacts in addition to high velocity impacts, and have been designed to mitigate both impact velocities.

Objective To compare the impact attenuation characteristics between ST and IH helmets, in both high and low velocity impacts.

Design Two-group experimental design.

Setting Impacts were performed in laboratory under controlled conditions. Helmets were impacted at two impact velocities (high and low; $4.5 \mathrm{~m} / \mathrm{s}$ and $2.4 \mathrm{~m} / \mathrm{s}$ respectively) and four impact locations (rear, rear boss, side and front boss). This was performed using a linear impactor device and the Hybrid III surrogate headform and neck.

Patients (or Participants) 5 different helmet models; 3 ST models and 2 IH models.

Interventions (or Assessment of Risk Factors) Assessment of ST and $\mathrm{IH}$ helmet impact attenuation under various conditions.

Main Outcome Measurements Peak linear and rotational acceleration; Head Injury Criterion (HIC) and Brain Injury Criterion (BrIC).

Results Between-groups ANOVA for linear [Low $F(1,27)=$ 10.7, $\mathrm{p}<0.05, \eta 2=0.284$; High $=\mathrm{F}(1,24)=5.8, \mathrm{p}<0.05$, $\eta 2=0.195]$ and rotational [Low $\mathrm{F}(1,27)=15.8, \mathrm{p}<0.05$, $\eta 2=0.370 ;$ High $=F(1,24)=8.1, p<0.05, \eta 2=0.251]$ accelerations yielded statistically significant differences with large effect sizes for all impact locations in both impact velocities. One-way between-helmet ANOVAs and post-hoc Bonferroni revealed impact attenuation performance hierarchy: IH 2 $>\mathrm{IH} 1>\mathrm{ST} 3>\mathrm{ST} 1>\mathrm{ST} 2$. Between-groups ANOVA revealed statistical differences for HIC [Low $\mathrm{F}(1,27)=14.1$, $\mathrm{p}<0.05, \eta 2=0.344 ;$ High $=\mathrm{F}(1,24)=7.6, \mathrm{p}<0.05, \eta 2=$ $0.241]$. BrIC results were mixed.

Conclusions Results suggest that these IH helmets are better at attenuating both impact velocities than this group of ST helmets. Interestingly, the largest effect sizes were observed in the low-velocity impacts.

\section{DO MOUTHGUARDS PREVENT ORO-FACIAL TRAUMA IN SPORT: A SYSTEMATIC REVIEW}

Peter Fine. UCL Eastman Dental Institute, London, UK

10.1136/bjsports-2021-IOC.312 
Background Sport can represent an important aspect of peoples' lives, for professional and recreational athletes. Some sports involve a high risk of orofacial trauma, particularly contact sports. Undoubtedly, mouthguards are considered by many as an effective solution in preventing injury.

Objective This systematic review aimed to measure the effectiveness of mouthguards in preventing oral-facial injuries.

Design Preferred Reporting Items for Systematic Reviews (PRISMA)

Setting Available electronic databases

Patients (or Participants) Contact sport athletes

Interventions (or Assessment of Risk Factors) MEDLINE and EMBASE electronic databases searches were supplemented by manual searching, of in-vivo studies examining prevention of orofacial injuries by protective devices that cover the teeth and surrounding soft tissues (mouthguards).

Main Outcome Measurements Included studies were assessed for methodological quality and bias. A meta-analysis was performed on data from selected studies, comparing the number of injuries for mouthguard users and non-users. Results are presented as forest plots and relative risks between different studies compared.

Results 1745 records were identified. Fifteen studies were eligible for inclusion in the qualitative synthesis, seven were included in the meta-analysis. The overall quality of studies was low, primarily due to a high degree of bias. The degree of heterogeneity was high throughout the studies affecting most of the variables such as sport, athletes' age, the definition of injury used and type of mouthguard. The meta-analysis showed that wearing a mouthguard was associated with a greater risk of orofacial injury when compared to non-users (overall RR 0.85\%, 95\% CI 0.78-0.93). However, the high degree of heterogeneity questions the validity of the summary estimate. $(\mathrm{I}$-squared $=91.2 \%$ ).

Conclusions There is insufficient evidence to support or refute the use of mouthguards to prevent orofacial injuries. Further research from high quality prospective cohort studies are needed to investigate the protective nature of mouthguards from orofacial injuries, when used in sports. The meta-analysis findings could be explained by athletes being more physical in the knowledge that they are protected.

\section{CAN A BEHAVIOUR CHANGE INTERVENTION IMPROVE ATHLETE ORAL HEALTH?}

Julie Gallagher, Paul Ashley, lan Needleman. UCL Eastman Dental Institute, London, UK

10.1136/bjsports-2021-IOC.313

Background Poor oral health of elite athletes is associated with negative performance impacts. There is a need for oral health promotion and prevention strategies that are effective within the elite sport environment

Objective To develop, implement and evaluate a pragmatic oral health promotion intervention based on contemporary behaviour change theory and informed by input from all stakeholders in elite sport.

Design Interrupted time-series intervention study.

Setting Three separate UK elite athlete-training centres.

Patients (or Participants) 62 athletes from two Olympic and one professional athlete training squads; 55 athletes completed the study.
Interventions (or Assessment of Risk Factors) Two levels: Level 1. Athletes and support team viewed combined one 10-minute presentation focussed on building motivation and three 90 -second information films. Level 2. Athletes alone received oral health screening, personalised advice and an oral health toolkit focussed on opportunity and capability. Follow-up of athletes at one and three months.

Main Outcome Measurements Impact on performance (OSTRC questionnaire), oral health knowledge (8-item questionnaire), gingival inflammation, self-reported oral health problems and oral hygiene routines.

Results At baseline, 41 (66.1\%) athletes had evidence of caries, 29 (46.8\%) evidence of erosion, mean bleeding score was 11.57 (8.11), $44(71 \%)$ were male and 58 (93.5\%) white British. Mean OSTRC score reduced from 8.73 (14.54) to 2.73 (11.31) $\mathrm{p}<0.001$. Mean knowledge score improved from 5.69 (1.59) to $6.93(1.32) \mathrm{p}<0.001$. Gingival bleeding score was unchanged. Self-reported oral health problems reduced. Athlete use of prescription fluoride toothpaste $(2800 \mathrm{ppm})$ increased from $8(12.9 \%)$ to $45(80.4 \%, \mathrm{p}<0.001)$.

Conclusions This behaviour change theory-based intervention was implemented within different elite sport environments. It was associated with a reduction in self-reported performance impacts, an increase in athlete oral health knowledge and enhanced oral health behaviour.

\section{EYE INJURIES IN SPORTS: AN UPDATE}

Thomas Henke, Gernot Jendrusch, Petra Platen. Ruhr-University Bochum, Dep. of Sports Medicine, Bochum, Germany

\subsection{6/bisports-2021-IOC.314}

Background About 3\% of all eye injuries happen during sports activities. About $25 \%$ of these eye injuries are so severe that they need ophthalmic treatment.

Objective Epidemiology of eye injuries in sports, risk of eye injuries in different sports.

Participants 2.392 eye injuries were analysed based on 221.273 sports injuries (club sports) that have been recorded by the sports injury database of the Ruhr-University Bochum and ARAG Sports Insurance between 1987 and 2017. In order to calculate the sports discipline related risk of eye injuries the frequency of eye injuries in one sports discipline has been qualified with regard to the frequency of all sports injuries that have been recorded in this sports discipline.

Results The average age of the 2.392 eye injured - this equals $1.08 \%$ of all sports injuries - was 31.9 (age range: 1 - 95 years). $78 \%$ of the injured persons were male, whereas $22 \%$ were female. About $19.7 \%$ had to stay in hospital, $13.9 \%$ needed surgery and $56.9 \%$ were incapable to work. On average, the injured had to pause their sport for 24.8 days. Blunt traumata, e.g. contusions, are dominant with over $50 \%$. These are often injuries caused by balls (e.g. in tennis or squash), rackets (e.g. in squash or ice hockey) or hand and elbow hits during tackles/tacklings (e.g. in handball or soccer). Assuming a mean risk of 1 for all sports, especially sports disciplines like squash (x8.4), badminton (x5.3), tennis (x4.8), but also water polo (x8.9) with swimming and grasping movements at eye level contain a significantly higher risk of eye injuries.

Conclusions Due to the special risk of eye injuries in squash the use of protective (sports) goggles is recommended from an 